\title{
Physics Based Design, the Future of Modeling and Simulation
}

T. S. Ericsen

This paper discusses the expanding role of modeling and simulation in the design and development of electrical power systems. The concepts of physics-based design and building blocks are introduced to show how complex systems may be simplified. However, the detail and complexity of tomorrow's systems are beyond today's tools. Computing power has increased to the point where physics-based design is possible. The aim of this paper is to discus the issues and opportunities for modeling and simulation in advanced system design.

Keywords: modeling, simulation, power electronics, power electronic building blocks, PEBB, electric power systems, advance marine electric power systems, naval electric power systems.

\section{Introduction}

Today, modeling and simulation are mostly used as analysis tools. However, systems with many power electronic components are emerging - driven by the need for power quality, availability, security, and efficiency. The detail and complexity of these "system-of-systems" exceeds the capability of today's rule-based design methods as reported by Ericsen [1]. Tomorrow's systems will require a relational and rational design process using modeling and simulation. The model becomes the specification. Paper documents cannot address the complexity of the next generation power electronic systems. Computer-aided design must become computer-based design.

Furthermore, two types of models are envisioned: requirement models and product models. Requirement models can be behavioral, empirical, and relational. Product models must be physics-based or functional to include the nature of the constituent materials and the methods of manufacture. Physics-based models enable one to predict the physics of failure, quantify risk as a function of known and unknown physics, and quantify cost as a function of materials and manufacturing.

Key parts of physics-based design are validation and incremental prototyping. Today's power electronic systems require a completed and commissioned system to validate the design. New power electronic concepts such as Power Electronic Building Blocks (PEBB) enable designers to avoid re-commissioning elements that have been proven in previous designs. A new power electronic system design, that uses the same PEBB elements as a previous design, need only validate the new application stresses and design elements. A physics-based design process can provide confidence levels and quantified risk to any degree of certainty. However, there is still a great cost to build and test the new elements and to characterize the system under new application stresses. If most of the elements in a system are new, the cost of validation can be great.

Real-time simulation, capable of running with real hardware as a Hardware-In-the-Loop (HIL) simulation, can reduce the cost of validating new complex system designs. HIL simulation enables parts of a converter (switches, phase-legs, and bridges) to be run with the rest of the converter that is emulated in a software simulation. Therefore, the whole converter does not need to be built to validate the design, only the new elements. In the same way, new converters could be simulated with an entire system emulated in a HIL simulation. A process of incremental prototyping can be developed that proceeds by calculating a minimum significant HIL experiment for a given design problem. Based on the results obtained, a new HIL experiment would be configured for the next minimum significant hardware validation. The process continues until an acceptable level of confidence is obtained from the incremental prototyping steps performed. In the case of completely new systems, these steps can be used to build one-of-a-kind prototypes that can be validated based on physics in a process where the building steps are quantified and confidence is established prior to each prototyping step.

\section{Complexity and detail}

Complex systems have the following attributes, as shown by [2]:

1. The more identical that a model must be to the actual system to yield predictable results, the more complex the system is.

2. Complex systems “ ... have emergence ... the behavior of a system is different from the aggregate behavior of the parts and knowledge of the behavior of the parts will not allow us to predict the behavior of the whole system."

3. "In systems that are 'complex', structure and control emanate or grow from the bottom up."

4. A system may have an enormous number of parts, but if these parts "interact only in a known, designed, and structured fashion, the system is not complex, although it may be big."

5. Although a physical system may not be complex, if humans are a part of the system, it becomes complex.

As our physical understanding increases, the details and the burdens on design increase. As the power of systems increases, lower order effects have substantial amounts of energy and that cannot be ignored. Converters in the 10 's of megawatt range can produce 100's of kilowatts of losses in the form of heat, electromagnetic interference (EMI), and mechanical vibration. These machines can make great heaters, radio transmitters, and noise amplifiers. Electric motors are also transducers and they act as speakers for noise. Electromagnetic interference can interact with communica- 
tions and other electronic machines and create system malfunctions and instabilities. Today's designer must take into account these interactions, as well as predict many other effects such as: system reliability, cost, environmental effects, health effects, and so on. These predictions span temporal ranges from microseconds, through days, to years.

Integration has become the workhorse of affordability and increased performance. With increased integration comes increased coupling and detail. For example, today's systems require designers to meet thermal, mechanical, electrical, and chemical requirements synergistically, rather than as independent design threads woven together at the end of the project. Digitally-controlled systems are multifunctional and the implications of functionality on the totality of application and environment must be understood before commissioning.

\section{Design cycle \& hierarchy}

In the classic design cycle, a requirement is given. A prototype must be synthesized. The prototype is then analyzed and the results compared to the requirement. If the requirement is satisfied, then the design cycle is complete and the product is produced. If the requirement is not satisfied, then the prototype is modified and analyzed. The results are again compared to the requirement. The cycle is repeated until the requirement is satisfied.

Analysis is well understood and the analysis problem is well posed. First, a solution exists. The solution is unique and stable - the solution depends continuously on the data.

Synthesis is ill-posed. There are many potential solutions not unique. The solution does depend continuously on the requirement. Often, the requirement is ill-defined. Synthesis is creative and uniquely human.

A larger design cycle continues in time where requirements drive new products and new products drive new requirements, see Fig. 1. Moreover, today's products must be able to meet complex cost, performance, and life tradeoffs. It is not enough to make something new and revolutionary. Products must meet market cost goals and not last longer than required. As noted above, modern integrated system design involves many sub-system designs and many engineering disciplines. This requires the talents of many designers from many different disciplines working collaboratively to design a system.

Extending this analogy a bit, let us say customers generate requirements and vendors create products in a design cycle. The vendor's prototype helps refine the customer's requirement. The customer's requirement drives the vendor's prototype. The customer's requirement is also a result of his own

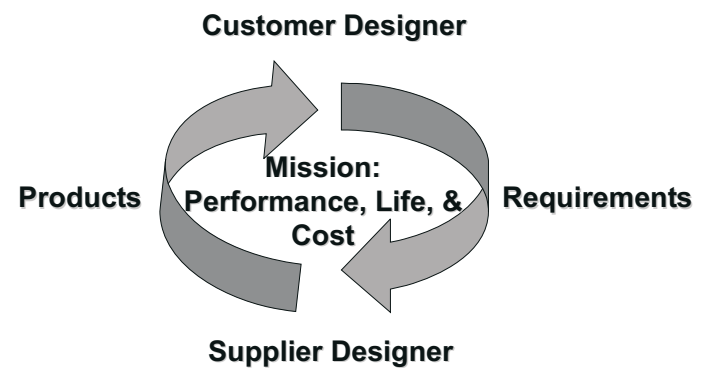

Fig. 1: Design cycle synthesis process to produce a prototype for a higher-level customer. One can envision a chain of customer/vendor design cycles extending from the basic materials, up through components, to systems. The product at highest system level may be a ship, a utility, or a city. There are always two designers at every level - customer and vendor designers. Finally, we need to take into account progressive integration processes that continually increase the coupling of thermal, mechanical, electrical, and chemical design threads. One can also imagine interlocking design cycles widening the design process to include these multi-discipline design teams.

At least two types of models are needed for this process models for the requirements and models for the products. The requirement model represents the top-down design point of view. The product model represents the bottom-up perspective. Since a requirement model needs to convey only performance and not specific solutions, it can be general or behavioral in nature. The product model conveys the manufacturer's specific solution to the requirement given; therefore, it must be physics-based or functional. There will be many possible product models for a single requirement model. If a vendor supplies a behavioral model in response to a requirement, the vendor is not clearly showing how material selection and the manufacturing processes employed yield a product, which will satisfy the necessary and sufficient conditions of the requirement.

\section{Simplification}

Up to this point, factors contributing to increased complexity and detail have been discussed. However, complex systems can be simplified by applying advanced technology. Using intelligent controllers and partitioning the system based on the physics of the materials, components, and methods of manufacture can produce building blocks, which allow systems to be designed, built, and operated in a rational predictable manner. For example, traditional electrical power systems are complex. Power is produced synchronously; $60 \mathrm{~Hz}$ (or $50 \mathrm{~Hz}$ ) sources supply power to $60 \mathrm{~Hz}$ loads. In equilibrium, the system is well-understood. However, when these systems are disturbed, the behavior becomes unpredictable and phenomena such as bifurcation can occur. In contrast, power electronic systems are not complex - theoretically. Power is produced, distributed, and consumed asynchronously. Power electronic machines such as converters, inverters, rectifiers, and motor controllers are active devices. When used intelligently in systems, they create known controlled and predictable states. Power electronic machines can have thousands of parts and many of these machines would be needed to simplify a system. Furthermore, power electronics add greatly to system size, weight, and cost. In this case, reducing complexity increases detail.

\section{Power electronic building blocks}

Power Electronics Building Blocks (PEBB) described in [3] and [4] exemplify the process of simplification. In 1994, the office Naval Research initiated the PEBB program to reduce the size, weight, and cost of power electronics so as to enable Advanced Electrical Power Systems, which enable Electric Warships with reduced manning, increased survivability, and 
increased power for electric propulsion, high power sensors, and high-power electric weapons as reported in [5] and by Ericsen [6]. The PEBB concept has transcended these initial objectives and is being refined by the IEEE Power Engineering Society, working group WGI8. PEBB-based systems can be found in many utility, marine, and industrial applications today.

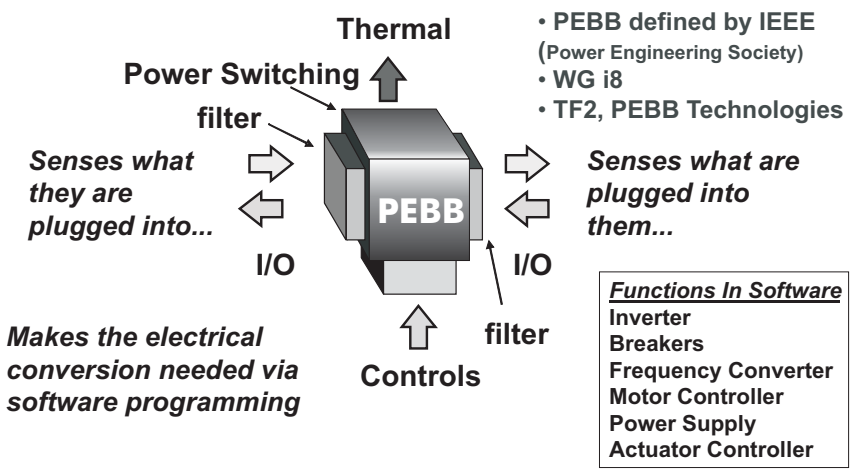

Fig. 2: Power electronic building blocks PEBB

Every power electronic block has input/output filters, power switching, control, and thermal management, as shown in Fig. 2. Ideally, a PEBB knows what it is plugged into and what is plugged into it, and makes appropriate connections. PEBB is a broad concept that incorporates progressive integration of power devices, gate drives, and other components into building blocks with defined functionality and interfaces - serving multiple applications; resulting in reduced cost, losses, weight, size, and engineering. The PEBB designer addresses details such as: device stresses, stray inductances, switching speed, losses, thermal management, and protection. The system engineer may apply PEBB in many diverse system applications without having to understand these details. Adoption of building blocks that can be used for mul- tiple applications results in high-volume production and in reductions to engineering effort, design testing, onsite installation, and maintenance work. The value of integration can be enhanced with standardization of interfaces.

Physics-based relationships are essential for the design of power electronics building blocks. High-energy levels in power applications require that all the natural conservation laws be obeyed - conservation of energy, voltage, current, torque, force, etc. Partitions based on physics are of primary importance. Since the PEBB is an active device, the temporal partitions and control interface definitions are as important as the PEBB blocks. The Universal Controller Architecture, another thrust of the IEEE WGI8 working group, partitions the control for a system built using PEBB. Standard control interfaces enable system control to be implemented top down. Furthermore, standard control interfaces enable PEBBs to be electronically tuned to meet custom performance requirements and to adapt to changing system applications, environmental, and mission operating conditions.

Power electronics is akin to microelectronics, part of silicon science, sand-based technology. Trends in microelectronics applications, i.e., computers, servers, controls, etc., have resulted in their assembly from functional building blocks with incredible reductions in cost and increases in performance. While very small power supplies have followed these trends, power electronics for higher power applications are just beginning this revolution.

Modular and hierarchical design principles are the cornerstones of the building block concept. The idea of open plug-and-play architecture is to build power systems in much the same way as personal computers. Ideally, PEBBs would be plugged into power electronics systems and operational settings would be made automatically. The system knows the PEBB capabilities, its manufacturer, and its operational requirements. Each $\mathrm{PEBB}$ maintains its own safe operating limits.

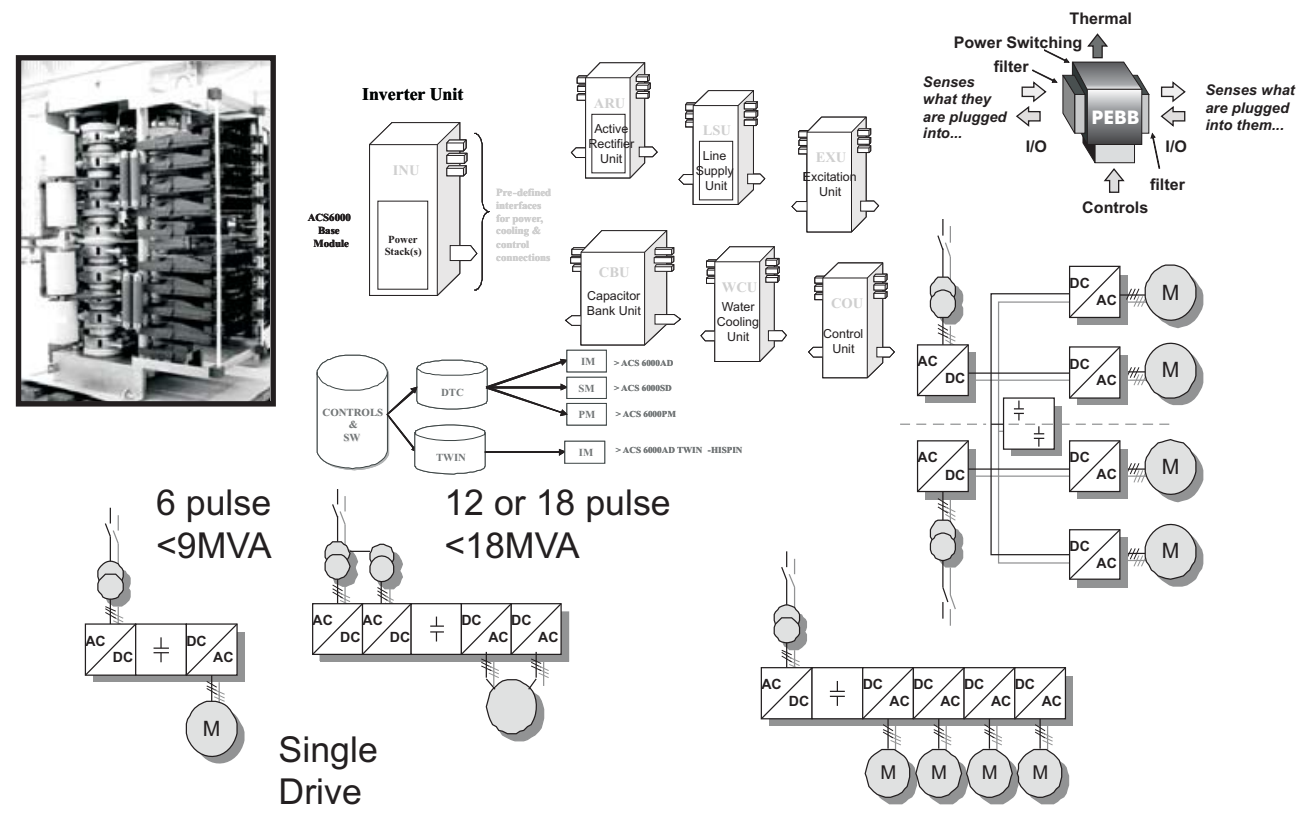

Fig. 3: 9 MVA PEBB, cabinets housing PEBBs, and systems using PEBBs, courtesy of ABB 
The U.S. Office of Naval Research (ONR) has funded several manufacturers to develop PEBBs for a broad range of applications. Some of these designs are now commercially available, although they may not be called PEBB products. One of these designs, the 9 MVA PEBB, is shown in the upper-left area of Fig. 3 [7]. This 9 MVA standard PEBB design is used in several marine, industrial, and utility applications. A smaller size 1 to 5 MVA PEBB has been commercialized for transportation, storage, marine and renewable energy source applications. In all cases, the PEBB is configured into standard cabinets; cabinets are selected and arranged as required; software programming is implemented for the specific application; and electronic tuning completes the system. Reductions in cost have been found in the range of $5 \times-10 \times$, and reduction in size and weight in the range of $2 \times-5 \times$. Progressive integration leading to reduced engineering effort and manufacturing cost has been the key.

PEBB concepts allow systems to be built rationally: based on pre-engineered building blocks, modeled and simulated with reduced detail, controlled from the top down, and the behavior of the system can be predicted based on the behavior of the building blocks that comprise it. The engineering effort and cost needed to produce a PEBB product is a tradeoff with the number of systems in which the PEBB can be applied. Therefore, the PEBB must be as generally applicable as possible to create the greatest return on investment.

Paradoxically, systems designed with PEBBs are potentially not complex; but the PEBB itself is most likely complex. Ideally, a PEBB can be designed based on several more layers of building blocks cascading down to the materials themselves. However, these building blocks have not been defined. Several projects have been tried and failed to produce an accurate PEBB model before building the PEBB. So far, designing and building the hardware PEBB takes much less time and cost than building a PEBB software model. In the future, it is reasonable to believe that these issues will be resolved. Additionally, the building block concept can be extended to other disciplines, as well. Actuator building blocks, mechanical building, and structural building blocks have all been proposed and studies initiated.

\section{Model is the specification, model is control, model is machine}

As can be seen from the previous discussions, the complexity and detail of modern systems is beyond paper specifications. Even when systems are simplified, detail increases dramatically. The detail is multi-disciplined and highly-coupled. Performance specifications are not specific enough to convey the engineering criteria of modern systems. Furthermore, performance specifications are a one-way street where the vendor is the only design agent. The model is only vehicle capable of conveying the engineering details needed and flexible enough to be used in true engineering design cycle. Moreover, the model is the only vehicle that has the potential for multi-physics relationships supporting integrated multi-discipline design. Thus, the model must become the specification and simulation the design medium for future systems.

The ability to program the control of a power electronic machine is a direct result of ONR's PEBB and Universal Controller Architecture programs. Many of today's controls are developed in software and implemented in microprocessors or programmable gate arrays. Most recently, algorithms developed in commercial software packages can be programmed into the targeted processors and tested directly from the software package, eliminating machine language programming and bench-top testing. In effect, the control developed on the desktop computer simulation software is the machine control. The model is the control. Fig. 4 shows the Universal Controller Architecture that describes the general partitioning for microprocessor controller platforms now used in many different industry applications. Analog to digital and digital to analog conversion occurs very close to the switches. The rest of the system is digital. The manufacturer can increase the production and reduce the cost of the hardware manufacture while tailoring the functionality to meet custom application requirements. It is not a big step to envision a future where different system functions can be implemented at different times by changing the equipment's software programming - the model becomes the machine.

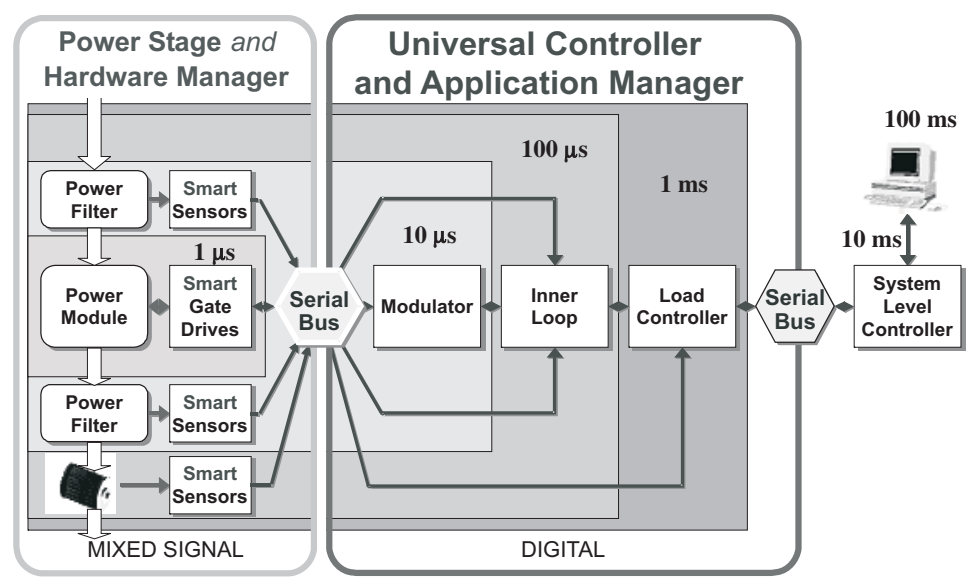

Fig. 4: Universal Controller Architecture, Power Engineering Society WGI8 Task Force 1 based on original work by and with permission from Deepak Divan 
If the model is to be the specification, then institutional support will be needed for activities such as software certification, standard models for calibration, standards for interfaces and protocols, and public libraries of physics-based models. Public libraries of standard physics-based models are essential to reduce duplication of non-proprietary effort and to gain the most from industry experience. The specification will be part of a legal agreement between the customer and vendor. Programming decisions, assumptions, and techniques can have a profound affect on the numerical accuracy and stability. One cannot qualify and quantify risk and the reliability of prediction, if the analysis cannot be certified.

The PEBB approach seeks to satisfy the necessary and sufficient conditions for the customer to analyze performance, while the relationships within the model can trace their roots directly to the underlying physics - as detailed as required for the questions asked. More work will be needed to achieve this goal. Yet, it is not clear if the model is one large multifaceted structure where the appropriate face is presented for the question asked, or if the model is a series of connected models where the right model is inserted in the simulation based on the question asked. In any case, the simulation environment must be able to change the models as needed without experts or additional expertise on the part of the inquirer.

If the model is the controller, then the simulation environment must be able to perform many forms of numerical analyses such as signal flow, conservation of energy, stability, and stress. Different solution methods can be connected by many means such as co-simulation, computational wrappers, and as a compiled model within another simulation. All of today's methods have different affects on computation speed and accuracy. Software designers need to rethink solvers and numerical processes to produce more robust simulation environments.

\section{Hardware in the loop}

If the model is to be the machine, the interface between hardware and software must be capable of real-time simulation. Simulations that interact with high frequency switching machines, such as inverters and converters, must be real time and high speed. For example, a HIL simulation could have the controller from a motor controller in a loop where the computer is supplying real-time signals to the controller to emulate the motor, power section of the motor controller, and the power source. The performance of the controller can be analyzed over many different operating conditions - some of which may too dangerous or costly to test otherwise. This is called Controller-In-The-Loop, CIL. A digital semiconductor curve tracer is an example of a Device-In-the-Loop, DIL. The curve tracer applies a preprogrammed stress to the device and the response of the device is recorded. Many different stresses can be applied and the device's characteristics can be mapped as a result. In this case, the HIL has everything the CIL has, plus programmable power supplies and possibly a heater, cooler, and other environmental stress controllers.

The next generation of HIL is the Digital Power Laboratory (DPL). If PEBBs can be configured and programmed to be motor controllers, propulsion drives, windmill controllers, and energy storage controllers, then one could use these PEBBs in a laboratory to emulate these loads. Likewise, power sources can be emulated with PEBBs. Furthermore, one does not need to duplicate every machine in a system to emulate the system. If a simulation of an entire system can be made, then an emulation of the system can be created at any point that replicates all of the artifacts of the energy and power at that point. Thermal, mechanical, and chemical aspects of the system at the point of interest can be included as well. Real power in real time is required at the interface between software simulation and hardware. Scaling methods can be used, if the underlying physics justify it. The primary limitations are bandwidth and power levels of the programmable power supplies.

\section{The future system design process}

It is time for system design, particularly Naval Ship design, to evolve. The pharmaceutical, microelectronics, and bio-medical industries, to name a few, have progressed to computer-based analysis and experimentation. A new computer is needed to combine human and machine intelligence and to fill the gap in advance system design. The scope of the design problem is daunting, bordering on unfathomable; but, complex systems can be simplified by physics-based partitioning and the use of intelligent active devices. Detail is increasing dramatically; but computational capability continues to grow dramatically, as well.

Notionally, the system design environment of the future will enable:

1. Human creativity - capable of synthesis as well as analysis which will enable software experimentation.

2. Customer and vendor design processes - models for requirements (top down) and products (bottom up).

3. Collaborative multidiscipline design -where many designers can work synergistically and simultaneously.

4. A relational design process - multi-discipline numerical processes allowing concurrent experimentation.

5. Building blocks - standard interfaces, model libraries, cellular and hierarchical design.

6. More robust and creative numerical solvers and environments.

7. HIL experiments.

8. Human and machine synergistic solvers - machines crunching millions of equations; humans watching for trends, cutting off unproductive threads, and creating leaps in solution space as inspiration and experience lead.

In the future, a new system project would begin with multi-discipline teams at their desktops logging into the design environment. Customer requirement models are up loaded. Libraries of physics-based models will be accessed to obtain needed elements. These models would be able to determine any new analysis conditions that go beyond the model's relevancy and assure the analysis limitations are established and known. The physics of the proposed system would first be divided into domains of known and unknown physics. If there are no new physics, no new application stresses, material, or components, then the design can be analyzed to assure compliance with customer requirements. If there is new physics, then risk and cost are directly related to the unknown or new physics. One will be able to quantify and 
qualify risk, time, and cost of the proposed system development - complete with confidence levels.

Computer experimentation begins with analysis of the domain of unknown physics. Polynomial neural nets, fuzzy logic, chaos, and other possibly new methods will enable researchers to understand the implications of the assumptions they make. It is very possible that these tools will be incorporated in the models themselves. These methods will enable researchers to find the details that humans tend to overlook, test the consistency of boundaries between known and unknown, and understand the logical extensions of physical assumptions.

At some point, analytical experiments are complete and a system hypothesis is formed. Hardware experiments are extracted from this hypothetical system by statistical analysis. Everything needed for the experiments is generated - test devices or machines, the test conditions, order of tests, relevant observations, etc. A Digital Power Laboratory (DPL) is configured directly from the results. After the DPL tests are performed, the problem is analyzed to determine the next steps - more unknowns or completed system design. If needed, a new hypothetical system is calculated based on these results. A new experimental series is executed. These cycles are continued until the customer and vendor are satisfied.

Although the above discussion is notional, two main points are clear. First, physical knowledge must be exploited to the fullest extent possible. If the components, materials, and algorithms have been applied previously, then these results can be used in the new design. The new design needs only to focus efforts on new elements and application conditions - not on redeveloping what is already known. Second, computer-based design and experimentation can achieve incremental prototyping. Physics-based modeling and simulation enables the design to be edited and validated without building and rebuilding hardware. With the concept of the DPL, new components and machines can be tested as if they were in the real system. As each unknown is validated, the system becomes incrementally validated.

\section{Conclusion}

In summary, the complexity and detail of modern systems are beyond today's modeling and simulation tools in six ways: 1) machine detail is beyond existing tools when design is taken in a conventional sense; 2) complexity is beyond tools when new and growing customer expectations are considered; and 3) complexity and detail exceed existing tools when entirety of the "system of systems" hierarchy is considered; 4) Today's modeling and simulation tools are primarily analysis tools - not designed for synthesis; 5) Today's tools assume there is only one designer, when there are many designers; 6) Tomorrow's electronically reconfigurable systems will need a new set of tools for design, control, and operation.
Furthermore, we are not producing enough engineers and scientists, worldwide, to address this complexity and increased detail. Human intelligence cannot expand to meet these challenges. Machine intelligence must be harnessed into a design environment with human intelligence, if we are to meet these future challenges. Finally, some of the key issues are: multidiscipline modeling and simulations; handling model order across vast temporal, spatial, and application ranges; manufacturing and material-based models, designing with uncertainty, the digital power laboratory for emulation and validation, and incremental prototyping.

\section{Acknowledgments}

I would like to acknowledge the extremely helpful discussions I have had with Roy Crosby, Narian Hingorani, and Albert Tucker during the writing of this paper.

\section{References}

[1] Ericsen, T.: "Future Navy Application of Wide Bandgap Power Semiconductor Devices." Proceedings of the IEEE, Vol. 90 (2002), No. 6, p. 1077-1082.

[2] Sage, A., Olson, S.: "Modeling and Simulation in Systems Engineering: Whither Simulation Based Acquisition?" SIM 2001 journal, Vol. 76 (2001), No. 2, p. 90-92.

[3] Panel Session on "Power Electronics Building Block Concepts" at the IEEE PES General Meeting in Toronto, July 2003.

[4] Document: Power Electronics Building Block Concepts. Under Preparation by IEEE Power Engineering Society Task Force 2 of Working Group I8 of Power Electronics Equipment Subcommittee of the Substation Committee. This Publication will be available soon.

[5] Ericsen, T., Tucker, A.: "Power Electronics Building Blocks and Potential Power Modulator Application." Proc. $23^{\text {rd }}$ Int. Power Modulator Symposium, Rancho Mirage, CA, June 1998, p. 12-15.

[6] Ericsen, T.: "Power Electronic Building Blocks - A Systematic Approach to Power Electronics." Proc. IEEE Power Engineering Society Summer Meeting, Vol. 2 (2000), p. 1216-1218.

\footnotetext{
Terry Sayre Ericsen

e-mail: ericset@onr.navy.mil
}

\section{ONR334}

Ship Hull, Mechanical, and Electrical Systems Science and Technology Division

Office of Naval Research

Arlington, Virginia, USA 